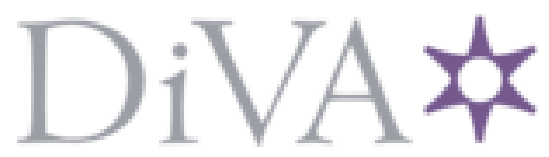

http://www.diva-portal.org

\title{
Postprint
}

This is the accepted version of a paper published in Journal of Colloid and Interface Science. This paper has been peer-reviewed but does not include the final publisher proof-corrections or journal pagination.

Citation for the original published paper (version of record):

Cui, Z., Edwards, K., Orellana, A., Bastiat, G., Benoit, J. et al. (2014)

Impact of interfacial cholesterol-anchored polyethylene glycol on sterol-rich non-phospholipid liposomes.

Journal of Colloid and Interface Science, 428: 111-120

http://dx.doi.org/10.1016/j.jcis.2014.04.031

Access to the published version may require subscription.

N.B. When citing this work, cite the original published paper.

Permanent link to this version:

http://urn.kb.se/resolve?urn=urn:nbn:se:uu:diva-223793 


\section{Impact of interfacial cholesterol-anchored polyethylene glycol on sterol-rich non-phospholipid liposomes}

Zhong-Kai Cui ${ }^{\mathrm{a}} \quad$ (zhongcai.cui@umontreal.ca), Katarina $\quad$ Edwards, ${ }^{\text {b }}$ (katarina.edwards@kemi.uu.se) Alejandro Nieto Orellana, ${ }^{\text {,dd }}$ (a.otein.o@gmail.com) Guillaume Bastiat, $^{\mathrm{c}, \mathrm{d}} \quad$ (guillaume.bastiat@,univ-angers.fr) Jean-Pierre Benoit, $\quad{ }^{\mathrm{c}, \mathrm{d}}$ (jean-pierre.benoit@univ-angers.fr) and Michel Lafleur ${ }^{\mathrm{a},{ }^{*}}$ (michel.lafleur@umontreal.ca)

${ }^{a}$ Department of Chemistry, Centre for Self-Assembled Chemical Structures (CSACS), Université de Montréal, C.P. 6128, Succ. Centre Ville, Montréal, Québec, Canada, H3C 3J7

${ }^{\mathrm{b}}$ Department of Chemistry - BMC, Uppsala University, Box 579, SE 751 23, Uppsala, Sweden. 'INSERM U1066, Micro et Nanomédecines Biomimétiques-MINT, Angers F-49933, France. dLUNAM Université, UMR-S1066, Angers F-49933, France.

*All correspondence should be sent to: Michel Lafleur

Telephone number: (514) 340-3205

Telefax number: (514) 343-7586

E-mail: michel.lafleur@umontreal.ca 


\section{Table of content (TOC) Graphic}

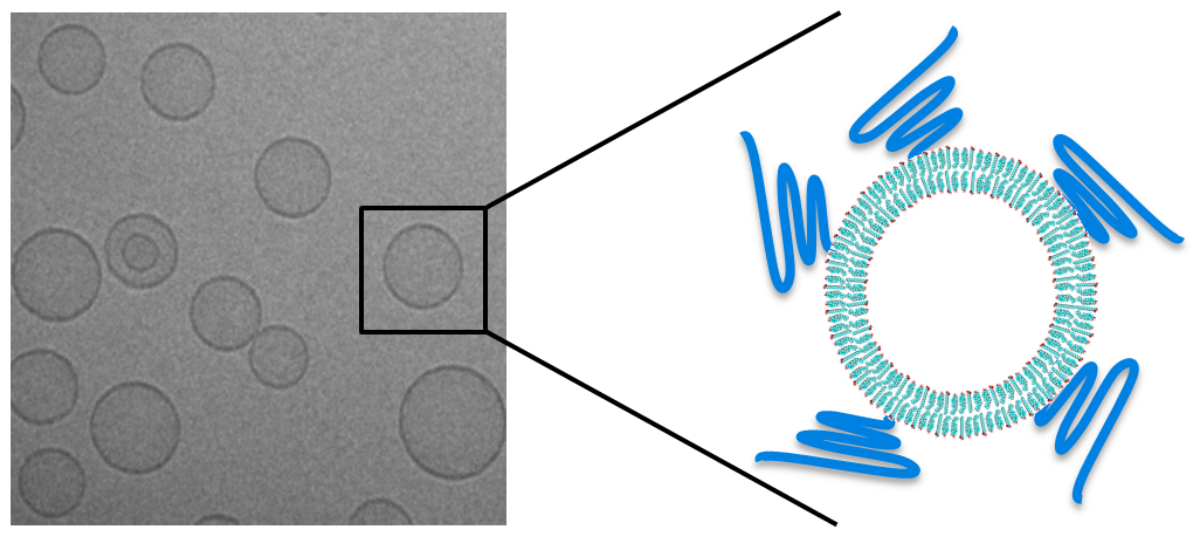

\section{Highlights}

- PEGylated liposomes strictly made from palmitic acid and cholesterol are created

- These non phospholipid liposomes display a very limited permeability

- Active loading of doxorubicin could be achieved in PA/Chol/PEG-Chol liposomes

- Interfacial PEG reduced the clearance of PA/Chol liposomes from the blood stream 


\begin{abstract}
Hypothesis

Liposomes made of single-chain amphiphiles and a large amount of sterols display several advantages including a limited permeability. In the present paper, we examine the possibility to prepare such non-phospholipid liposomes with interfacial polyethylene glycol (PEG) in order to improve their circulation in the blood stream. Cholesterol (Chol) was chosen as the PEG anchor.

\section{Experiments}

The phase behavior of mixtures of palmitic acid (PA) and cholesterol including various proportions of PEGylated cholesterol (PEG-Chol) was characterized. In conditions leading to the formation of fluid bilayers, properties of the resulting liposomes were assessed.

\title{
Findings
}

Up to $20 \mathrm{~mol} \%$ of PEGylated cholesterol could be introduced without significant perturbations in fluid bilayers made of PA and cholesterol. With $10 \mathrm{~mol} \%$ PEG-Chol, PA/Chol/PEG-Chol liposomes showed a very limited permeability to calcein and doxorubicin. Doxorubicin could be actively loaded in PA/Chol/PEG-Chol liposomes with a high drug loading efficiency and a high drug to lipid ratio. Pharmaco-kinetic experiments in rats indicated that interfacial PEG reduced the clearance of $\mathrm{PA} / \mathrm{Chol}$ liposomes compared to the naked ones. However the lifetime of these non-phospholipid liposomes in the blood circulation was considerably shorter than that observed for control PEGylated phospholipid liposomes, a phenomenon associated with the negative interfacial charge of the PA/Chol/PEG-Chol liposomes.

Key Words: Liposomes; cholesterol; palmitic acid; polyethylene glycol; nanovectors; permeability; pharmaco-kinetics; active loading. 


\begin{abstract}
Abbreviations:mononuclear phagocyte system (MPS); polyethylene glycol (PEG); distearoylphosphatidylethanolamine (DSPE); PEG-phosphatidylethanolamine (PEG-PE); palmitic acid (PA); cholesterol (Chol); PEGylated cholesterol (PEG-Chol); large unilamellar vesicles (LUVs); doxorubicin (DOX); tris(hydroxymethyl)aminomethane (TRIS); 2-[N-morpholino]ethanesulfonic acid (MES); ethylenediaminetetraacetic acid (EDTA); perdeuterated palmitic acid $\left(\mathrm{PA}-d_{31}\right) ; 1,1^{\prime}$-dioctadecyl-3,3,3',3'-tetramethylindodicarbocyanine perchlorate (DiD); Multilamellar Vesicles (MLVs); dynamic light scattering (DLS); cryogenic transmission electron microscopy (Cryo-TEM)
\end{abstract}




\section{Introduction}

Liposomes are biocompatible, biodegradable, biologically inert, weakly immunogenic, and they display several advantages for drug vectorization. ${ }^{1-2}$ They can provide a slow release that expands the period during which the drug level remains in the therapeutic window, they can target, passively or actively, specific sites, and they can reduce the acute toxicity of drugs. One of the limitations of liposomes used as drug carriers for intravenous administration, however, is their rapid capture and removal from the blood circulation by the mononuclear phagocyte system (MPS), leading to their accumulation in the liver and the spleen. Because it leads to a tighter chain packing, the incorporation of cholesterol in liposomes reduces the transfer of lipids to high-density lipoproteins and prevents blood-protein binding, extending the liposome circulation

lifetime in the blood stream. ${ }^{3-5}$ A major breakthrough for improving circulation time and reducing MPS uptake was the introduction of gangliosides and sialic acid derivatives, such as monosialoganglioside $(\mathrm{GM} 1)^{6-7}$ at the water/liposome interface. A similar effect was observed with interfacial polyethylene glycol (PEG) ${ }^{8-10}$ This protective effect has been associated with the steric stabilization of liposomes that inhibits the uptake by MPS and prolongs the liposome circulation lifetime. ${ }^{6,8-9,11}$ The origin of this protection is still not fully understood but it has been proposed that the reduced binding of plasma proteins, including potential opsonizing factors, the limitation of direct interactions with cells of the reticuloendothelial system, and the inhibition of liposome-liposome aggregation could contribute to the reduced clearance of PEGylated liposomes ${ }^{12-18}$.

PEG is an appealing macromolecule for obtaining sterically-stabilized liposomes as it displays biocompatibility, low toxicity, low immunogenicity and antigenicity, and good excretion kinetics. ${ }^{2}$ Furthermore, its molecular weight can be easily modulated and it is relatively cheap to produce. It has been shown that PEG with a molecular weight between 1000, and 5000, anchored 
on bilayers with a phosphatidylethanolamine (PE) moiety displays a good miscibility with phospholipids; up to $\sim 10 \mathrm{~mol} \%$ PEG could be grafted without observing phase separation or micelle formation. ${ }^{13,19}$ It was shown that improved circulation times of liposomes in the blood stream were obtained for liposomes with 2-10 mol\% of interfacial PEG with a molecular weight between 350 and 2000 (anchored with distearoylphosphatidylethanolamine (DSPE) in these studies). ${ }^{12,} 16$ The protection of liposomes against phagocytosis was also improved with introducing PEG with a molecular weight of 2000 at its interface. ${ }^{9}$ An increase of the PEG molecular weight to 5000 had no effect on half-life time of liposomes in the blood stream or, in some reports, slightly decreased it. ${ }^{9}$

The anchoring of PEG at the liposome interface is achieved by covalently attaching PEG to a phospholipid or a sterol. Because of the reactivity of the amine group, PEG-phosphatidylethanolamine (PEG-PE) is commonly used. ${ }^{2}$ PEG-coupled with ceramide has also been synthesized as a neutral alternative. ${ }^{20}$ Ishiwata and coll. ${ }^{21}$ used a sterol anchored PEG for modifying liposome surface. PEGylated cholesteryl ether led to an increased liposome circulation time in the blood stream similar to that previously obtained with PEG-DSPE. ${ }^{21-23}$ PEG cholesteryl ester was also synthesized and incorporated in phospholipid liposomes and niosomes to achieve long circulation lifetime. ${ }^{23-24}$ As a hydrophobic anchor for PEG, cholesterol is an alternative that presents distinct advantages. First, the chain ordering effects of cholesterol lead to less permeable bilayers. Second, it is neutral and this property provides a reduced binding of plasma proteins to liposomes. Third, cholesterol is chemically stable, a useful feature regarding storage.

It has been shown that it is possible to form non-phospholipid liposomes with single-chain amphiphiles and sterols - for a review, see 25-26. The binary mixtures that could successfully form liposomes include negatively charged mixtures of unprotonated palmitic acid 
(PA) or its analogues with cholesterol (Chol) or some other sterols. ${ }^{27-29}$ A distinct feature of these non-phospholipid liposomes is their high sterol content, which is between 50 and $70 \mathrm{~mol} \%$. This peculiar composition leads to a permeability that is drastically limited compared to traditional phospholipid liposomes. For example, it was found that about $70 \%$ of entrapped calcein, a negatively charged fluorophore, was still encapsulated after 1.5 years. ${ }^{28}$ In addition, these liposomes are $\mathrm{pH}$ sensitive when they include a fatty acid or an alkylated primary amine as the single-chain amphiphile. The pH-triggered release could be fine-tuned by selecting a fatty acid with an appropriate $\mathrm{pKa}^{28}$

These non-phospholipid sterol-rich liposomes display an interesting potential as drug nanovectors. Their constituents are significantly more stable from a chemical point of view in aqueous suspension than phospholipids. Because of their very limited permeability, they could present a valuable alternative for drug encapsulation especially in the cases of challenging drugs that leak rapidly from conventional phospholipid liposomes such as $5-\mathrm{FU} .{ }^{30} \mathrm{In}$ these cases, it has been shown that it was difficult to retain the drug inside phospholipid-based liposomes, preventing the development of liposomal formulations for intravenous therapy displaying extended blood circulation lifetime and improved therapeutic activities. The restricted leakage observed for liposomes prepared with single-chain amphiphiles and sterols could therefore be advantageous in that context. In order to obtain a sustained drug release in parenteral delivery, nanovectors should also display a long circulation lifetime in the blood stream. Preliminary pharmaco-kinetics studies showed that, despite the very tight lipid chain packing that could limit protein adsorption, the sterol-rich $\mathrm{PA} / \mathrm{Chol}$ liposomes were cleared very rapidly (less than 30 minutes) from the blood stream in rats (unpublished results). In the present study, we examined the possibility of introducing PEGylated cholesterol (PEG-Chol) in non-phospholipid liposomes made of PA/Chol mixtures in order to combine the incomparably low permeability of those with improved blood circulation lifetime. We determined the impact of the incorporation of PEG-Chol 
on the stability of these unusual bilayers using deuterium and pulsed-field-gradient ${ }^{1} \mathrm{H} \mathrm{NMR}$, as well as cryo-transmission electron microscopy. We established the amount of PEG-Chol that could be included in the non-phospholipid bilayers without significant perturbation. We examined also the possibility to extrude the ternary mixtures to form large unilamellar vesicles (LUVs) and characterized the stability, the permeability and the $\mathrm{pH}$ sensitivity of the resulting liposomes. These findings provided us with a more detailed understanding of the influence of the hydration of the liposome interface on the stability of these self-assemblies. The present study also displays the feasibility of actively loading a drug in these non-phospholipid vesicles. Active loading is an advantageous way to obtain high drug loading efficiency and high drug to lipid ratios, two parameters presenting considerable benefit for therapeutic purposes and the pharmaceutical industries. Because these liposomes with high sterol content are very impermeable, active loading may present a specific challenge. We have therefore investigated whether LUVs prepared from PA/Chol/PEG-Chol mixtures could be actively loaded with doxorubicin (DOX), a broad-spectrum anticancer drug. Liposomal DOX has been shown to have improved therapeutic index and decreased toxicity. ${ }^{31-32}$ It is somehow a benchmark regarding drug active loading. DOX is an amphipathic weak base that can be actively loaded into the aqueous compartment of phospholipid liposomes through ammonium sulphate gradient with a high drug loading efficiency. The ammonium sulphate gradient approach does not require the preparation of liposomes in acidic $\mathrm{pH}$, nor to alkalinize the extraliposomal aqueous phase, by contrast with other chemical approaches used for active loading. ${ }^{33}$ Finally, we conducted pharmaco-kinetic experiments in rats in order to determine the effect of interfacial PEG on the blood circulation lifetime and the biodistribution of PA/Chol/PEG-Chol liposomes. The pharmaco-kinetics was examined using DiD, a fluorescent lipophilic probe that labels the LUV walls and has, in general, limited transfer from labeled to unlabeled membranes. ${ }^{34}$ 


\section{Materials and Methods}

Materials. Hydroxypolyethyleneglycol cholesteryl ether, with PEG MW of 2000, (PEG-Chol) was purchased from NOF Corporation (Tokyo, Japan). Cholesterol (>99\%), palmitic acid (99\%), tris(hydroxymethyl)aminomethane (TRIS) (99\%), 2-[N-morpholino]ethanesulfonic acid (MES) (>99\%), ethylenediaminetetraacetic acid (EDTA) (99\%), $\mathrm{NaCl}(>99 \%)$, Triton X-100 (99\%), and deuterium-depleted water ( $>99.99 \%)$ were supplied by Sigma Chemical Co. (St. Louis, MO, USA). Perdeuterated palmitic acid $\left(\mathrm{PA}-d_{31}\right)(98.9 \%)$ and deuterium oxide $(>99 \%$, $\mathrm{D}_{2} \mathrm{O}$ ) were from CDN Isotopes (Pointe-Claire, QC, Canada). Calcein (high purity), and 1,1'-dioctadecyl-3,3,3',3'-tetramethylindodicarbocyanine perchlorate (DiD) were obtained from Invitrogen (Burlington, ON, Canada). Sephadex G-50 Medium was purchased from Pharmacia (Uppsala, Sweden). Methanol (spectrograde) and benzene (high purity) were obtained from A\&C American Chemicals Ltd. (Montreal, Qc, Canada), and BDH Inc. (Toronto, ON, Canada), respectively. DOX (hydrochloride salt, $>99 \%$ ) was obtained from LC Laboratories (Woburn, MA, USA). Ammonium sulfate (>99.0\%) and Dowex ${ }^{\circledR} 50 \mathrm{WX} 4$ were supplied by Alfa Aesar (Ward Hill, USA). Dowex ${ }^{\circledR} 50 \mathrm{WX} 4$ was obtained as a strong acidic cation exchange resin and converted to the sodium form. All solvents and products were used without further purification.

Multilamellar and Large Unilamellar Vesicle Preparation. Mixtures of PA/Chol/PEG-Chol were prepared by dissolving weighed amounts of the solid chemicals in a mixture of benzene/methanol 90/10 (v/v). The solution were then frozen in liquid nitrogen and lyophilized for at least $16 \mathrm{~h}$ to allow complete sublimation of the organic solvent. For the NMR experiments, PA was replaced by PA- $d_{31}$. In order to form Multilamellar Vesicles (MLVs), the freeze-dried lipid mixtures were hydrated with a buffer. The final lipid concentration was 30

$\mathrm{mg} / \mathrm{mL}$ unless otherwise stated. The suspensions were subjected to five cycles of freezing-and-thawing (from liquid nitrogen temperature to $\sim 70{ }^{\circ} \mathrm{C}$ ) and vortexed between 
successive cycles to ensure a good hydration of the samples. The LUVs were obtained by extruding MLVs using a handheld Liposofast extruder (Avestin, Ottawa, Canada). Typically the dispersions were passed 15 times through two stacked polycarbonate filters at room temperature.

${ }^{2}$ H NMR Spectroscopy. MLVs were prepared in a MES/TRIS buffer (TRIS $50 \mathrm{mM}$, MES $50 \mathrm{mM}, \mathrm{NaCl} 10 \mathrm{mM}$, EDTA $5 \mathrm{mM}$ ), providing a buffered range between $\mathrm{pH} 5$ and 9 . The MES/TRIS buffer was prepared with deuterium depleted water for these experiments. The samples were then transferred into homemade Teflon holders. The ${ }^{2} \mathrm{H}$ NMR spectra were recorded on a Bruker AV-600 spectrometer, using a Bruker static probe equipped with a $5 \mathrm{~mm}$ coil. A quadrupolar echo sequence was used with a $90^{\circ}$ pulse of $2.9 \mu$ s and an interpulse delay of $35 \mu$ s. The recycling time was $60 \mathrm{~s}$. In absence of a slow-relaxation component, namely a solid phase, the recycling delay was reduced to 0.3 s. Typically 4000 FIDs were co-added. The temperature was regulated using a Bruker VT-3000 controller. The proportion of the isotropic phase in the ${ }^{2} \mathrm{H}$ NMR spectra showing the co-existence of the lo lamellar phase and an isotropic phase could be estimated from the difference in the spectrum areas prior to and after the elimination of the narrow central peak.

${ }^{1}$ H NMR Diffusion Experiments. In order to assess the inclusion of PEG-Chol in the liposomes, the diffusion coefficient of PEG was determined on LUVs prepared from different compositions, using ${ }^{1} \mathrm{H}$ pulsed-field-gradient NMR. These LUVs were obtained by extruding through two stacked polycarbonate filters (200 nm-pore size) an MLV suspension prepared in a $\mathrm{D}_{2} \mathrm{O}$-based MES/TRIS buffer. ${ }^{1} \mathrm{H}$ NMR diffusion experiments were performed on a Bruker AV-400 spectrometer operating at $400 \mathrm{MHz}$ for ${ }^{1} \mathrm{H}$. The diffusion measurements were carried out at $25{ }^{\circ} \mathrm{C}$ using a Bruker diffusion probe (Diff60) equipped with a ${ }^{1} \mathrm{H} /{ }^{2} \mathrm{H}$ 5-mm coil. This probe includes one gradient coil, along the $z$ axis, capable of delivering magnetic field gradients up to $2900 \mathrm{G} / \mathrm{cm}$. The gradient strength was calibrated from the diffusion coefficient of $1 \% \mathrm{H}_{2} \mathrm{O}$ in a 
$\mathrm{D}_{2} \mathrm{O}$ sample. The diffusion coefficients were measured using the stimulated echo pulse sequence $^{35}$. Trapezoidal gradient pulses $(\delta)$ of $1.50 \mathrm{~ms}$ and interpulse delays $(\Delta)$ of $100 \mathrm{~ms}$ were applied. Typically, 32 scans were co-added for a given gradient strength and 32 gradient magnitudes were used for each attenuation curve. The sample temperature was controlled using the gradient coil cooling unit, and was calibrated using ethylene glycol. The diffusion coefficients were obtained by fitting the variation of the echo intensity as a function of the gradient strength (G) using the following equation: $\quad \ln \left(S / S_{0}\right)=-(G \gamma \delta)^{2} D(\Delta-\delta / 3)$

\section{(1)}

where $S$ and $S_{0}$ are the integrated echo intensities with and without a field gradient and $\gamma$ the gyromagnetic ratio of protons. All the curves could be well fitted with this single-diffusion-coefficient model.

LUVs Characterization. The hydrodynamic diameters of the LUVs were measured at 25 ${ }^{\circ} \mathrm{C}$ by dynamic light scattering (DLS) using a Malvern nanosizer. The curve fittings of correlation functions were performed using an exponential fit (CONTIN approach) for size (hydrodynamic diameter) determination. The scattering light intensity was adjusted by diluting the dispersions with the MES/TRIS buffer.

The conditions for the cryogenic transmission electron microscopy (Cryo-TEM) technique are detailed elsewhere. ${ }^{36}$ Briefly, an aliquot of a LUV sample $(\sim 1 \mu \mathrm{L})$ was deposited on a copper grid covered with a carbon-reinforced holey polymer film, dabbed with a filter paper to form a thin film $(10-500 \mathrm{~nm})$ and incubated in a custom-built climate chamber at $25{ }^{\circ} \mathrm{C}$ and $>99 \%$ relative humidity. The grid was then plunged into liquid ethane held at a temperature just above its freezing point $\left(-183^{\circ} \mathrm{C}\right)$. The vitrified sample was kept below $-165{ }^{\circ} \mathrm{C}$ and protected from ambient conditions during the transfer from the preparation chamber to the microscope as well as during the data acquisition. The micrographs were obtained using a Zeiss 
EM 902A Transmission Electron Microscope (Carl Zeiss NTS, Oberkochen, Germany), in the zero loss bright-field mode, with an accelerating voltage of $80 \mathrm{kV}$. The digital images were recorded under a low dose conditions with a BioVision Pro-SM slow scan CCD camera (Proscan GmbH, Scheuring, Germany). Their contrast was enhanced using an underfocus of 1-2 $\mu \mathrm{m}$.

Permeability Measurements. The permeability of the LUVs was measured using a standard procedure based on the self-quenching property of calcein at high concentration. ${ }^{28,37}$ Briefly, LUVs loaded with the fluorophore were prepared from PA/Chol/PEG-Chol mixtures hydrated with a MES/TRIS buffer, $\mathrm{pH} 8.4$, containing $80 \mathrm{mM}$ of calcein; the resulting MLV suspension was extruded, using filters with $100 \mathrm{~nm}$-pore diameter. The calcein-containing LUVs were separated from free calcein by gel permeation chromatography, using Sephadex G-50 Medium gel (column diameter: $1.5 \mathrm{~cm}$, length: $25 \mathrm{~cm}$ ), equilibrated with an iso-osmotic MES/TRIS buffer (MES $50 \mathrm{mM}$, TRIS $50 \mathrm{mM}, \mathrm{NaCl} 130 \mathrm{mM}$, EDTA $5 \mathrm{mM}$, pH 8.4). The collected vesicle fraction was diluted 100 times with buffers having different $\mathrm{pH}$ to obtain a final fatty acid concentration of about $20 \mu \mathrm{M}$. This dilution step was set as time $=0$. These stock LUV suspensions were then incubated at room temperature.

To study the passive leakage (external and internal $\mathrm{pH}$ of 8.4), the calcein fluorescence intensity was measured from an aliquot of the stock LUV suspension freshly isolated by gel permeation chromatography, prior $\left(\mathrm{I}_{\mathrm{i}}\right)$ and after $\left(\mathrm{I}_{\mathrm{i}+\mathrm{T}}\right)$ the addition of Triton X-100 $(10 \mu \mathrm{L}$ of a 10 $(\mathrm{v} / \mathrm{v}) \%$ solution prepared in the MES/TRIS buffer). The low fluorescence intensity measured prior to the detergent addition was associated to calcein that was initially completely encapsulated whereas the fluorescence intensity measured after addition of Triton X-100 corresponded to the complete release of entrapped calcein.

After a given incubation time, the calcein fluorescence intensity was measured on another 
aliquot of the same stock LUV suspension before $\left(\mathrm{I}_{\mathrm{f}}\right)$ and after $\left(\mathrm{I}_{\mathrm{f}+\mathrm{T}}\right)$ the addition of Triton X-100. The percentage of encapsulated calcein at that time in the LUVs was calculated according to:

$$
\% \text { of encapsulated calcein }=\left(\frac{\left(I_{f+T}-I_{f}\right) / I_{f+T}}{\left(I_{i+T}-I_{i}\right) / I_{i+T}}\right) \times 100
$$

The $\%$ of release corresponded to (100 - \% of encapsulated calcein).

The pH-triggered leakage of calcein was also examined. The percentage of released calcein was calculated using Equation 2: $\mathrm{I}_{\mathrm{i}}$ and $\mathrm{I}_{\mathrm{i}+\mathrm{T}}$ were measured at $\mathrm{pH} 8.4$, the initial $\mathrm{pH}$, before and after the addition of Triton X-100 respectively, whereas $\mathrm{I}_{\mathrm{f}}$ and $\mathrm{I}_{\mathrm{f}+\mathrm{T}}$ were obtained on an aliquot at a modified $\mathrm{pH}$, before and after the addition of Triton $\mathrm{X}-100$ respectively. The $\mathrm{pH}$ effect was examined for an increase as well as a decrease in $\mathrm{pH}$. Calcein fluorescence intensity was relatively constant over the investigated $\mathrm{pH}$ range. ${ }^{37}$

The fluorescence intensities were recorded using a Photon Technology International spectrofluorometer. The excitation and emission wavelengths were 490 and $513 \mathrm{~nm}$ respectively and the band path widths were set to 1.0 and $1.6 \mathrm{~nm}$ for the excitation and emission monochromators, respectively.

DOX Active Loading Experiments. Active loading of doxorubicin was carried out using an ammonium sulfate gradient. ${ }^{33} \mathrm{PA} / \mathrm{Chol} / \mathrm{PEG}-\mathrm{Chol}$ solid mixtures were hydrated with an ammonium sulfate solution $(120 \mathrm{mM})$. The PA/Chol/PEG-Chol LUVs (extruded using filters with 100 -nm diameter pores) were prepared as described above. The ammonium sulfate gradient was created by gel permeation chromatography, using Sephadex G-50 Medium gel (column diameter: $1.5 \mathrm{~cm}$, length: $25 \mathrm{~cm})$, equilibrated with an iso-osmotic $\mathrm{NaCl}$ solution $(150 \mathrm{mM})$. An aliquot of a DOX solution ( $5 \mathrm{mM}$, prepared in water containing $150 \mathrm{mM} \mathrm{NaCl}$ ) was added to the collected vesicles $(\sim 3 \mathrm{mg}$ of lipids/mL) in order to have a DOX/lipid molar ratio of 1/15. The LUVs suspensions were incubated at $\sim 70{ }^{\circ} \mathrm{C}$ for 24 hours. In order to determine the drug loading 
efficiency, the LUV suspensions incubated with DOX were diluted 100-folds with the $\mathrm{NaCl}$ solution. Converted Dowex ${ }^{\circledR} 50 \mathrm{WX} 4$ resin was added to an aliquot to remove the free DOX by complexation. The sample was centrifuged at $4000 \mathrm{rpm}$ for $3 \mathrm{~min}$ and DOX absorbance at 480 $\mathrm{nm}$ was measured. The absorbance was also measured for an aliquot without resin to obtain the total amount of DOX. The release of liposome-entrapped DOX was also characterized by UV-vis spectroscopy using the resin treatment to eliminate the released drug. HPLC-MS analysis was carried out on LUV aliquots treated with the resin in order to quantify the drug to lipid ratio.

Pharmacokinetics and biodistribution. Sprague-Dawley rats (300-350 g) (Charles River, L'Arbresle, France) were housed and maintained at the University animal facility (SCAHU). All the animal experiments were performed in agreement with the EEC guidelines, and the "Principles of Laboratory Animal Care" (NIH Publication No. 86-23, revised 1985), and with the agreement of Comité d'éthique pour l'expérimentation animale des Pays-de-la-Loire (authorization CEEA; 2012-180). Rats ( $n=8$ for each group) were anesthetized (with isoflurane). PA/Chol, PA/Chol/PEG-Chol or DSPC/Chol/PEG-DSPE LUVs in phosphate buffer (137 mM $\mathrm{NaCl}, 2.7 \mathrm{mM} \mathrm{KCl}, 10 \mathrm{mM} \mathrm{Na} 2 \mathrm{HPO}_{4}, 1.76 \mathrm{mM} \mathrm{KH}_{2} \mathrm{PO}_{4}, \mathrm{pH}$ 7.4), were prepared as described above; the final lipid concentration was $7 \mathrm{mg}$ of lipids/mL. DiD, used to label the LUV walls, was added to the organic lipid solution, before the freeze-drying step, to obtain a final DiD/lipid ratio of $2(\mathrm{w} / \mathrm{w}) \%$. The LUV size and zeta potential were not affected by the presence of DiD, confirming that the fluorophore, at that concentration, did not affect the assembly. ${ }^{34}$ A LUV aliquot of $400 \mu \mathrm{L}$ was intravenously delivered (caudal veins) to each rat. At various time points, from $5 \mathrm{~min}$ to $24 \mathrm{~h}$ following the injection, blood samples (about $400 \mu \mathrm{L}$ ) were collected. The rats were sacrificed at the last time point, and liver, spleen, heart, lungs, brain, and kidneys were harvested.

Calibration curves were obtained by mixing liposomes with fresh blood at various concentrations and by measuring the fluorescence using a microplate reader Fluoroscan Ascent ${ }^{\circledR}$ 
(Labsystems SA, Cergy-Pontoise, France). Excitation and emission wavelengths were fixed at 646 and $678 \mathrm{~nm}$, respectively. The liposome concentrations in blood at the various time points were calculated on the assumption that blood represents $7.5 \%$ of rat body weight ${ }^{38}$. Organ fluorescence imaging and analysis were performed using a fluorescence imaging system (CRI Maestro $^{\mathrm{TM}}$, Woburn, MA, USA). The photon average count between 630 and $600 \mathrm{~nm}$ was obtained from each organ, using a time exposition of $500 \mathrm{~ms}$. The reported values correspond to the mean values \pm standard deviations obtained for $\mathrm{n}=8$. The statistical differences were established using the Mann Whitney test.

\section{Results}

Figure 1 presents ${ }^{2} \mathrm{H}$ NMR spectra of PA- $d_{31} / \mathrm{Chol} / \mathrm{PEG}-\mathrm{Chol}$ mixtures of various compositions: the PA- $d_{31} /$ sterol molar ratio was always $30 / 70$ and cholesterol was progressively substituted by PEG-Chol. This set of data provided us with the proportion of PEG-Chol that could be incorporated in the mixture while keeping a fluid lamellar arrangement. The spectra of PA- $d_{31} /$ Chol (30/70) mixture, $\mathrm{pH} 8.4$ (Figure 1A, top row), were essentially characteristic of a fluid lamellar phase. They corresponded to several overlapping powder patterns with different quadrupolar splittings, associated with the gradient of orientational order existing along the acyl chains. ${ }^{39-40}$ The quadrupolar splitting of the outermost doublet of the spectrum recorded at $25{ }^{\circ} \mathrm{C}$ was $55 \mathrm{kHz}$; this large value is typical of lipids in the lo phase. ${ }^{39-40}$ These spectra indicated that the lo phase formed by this mixture was stable over the whole investigated temperature range, in agreement with previous results. ${ }^{39}$ Actually, temperature variations between 25 and $60{ }^{\circ} \mathrm{C}$ had limited effect on these systems, the value of the quadrupolar splitting of the outermost doublet decreasing by only $\sim 5 \mathrm{kHz}$. Once $20 \%$ or more of PEG-Chol was introduced in the liposomes, a narrow peak centered at 0 appeared, indicating the formation of an isotropic phase. 


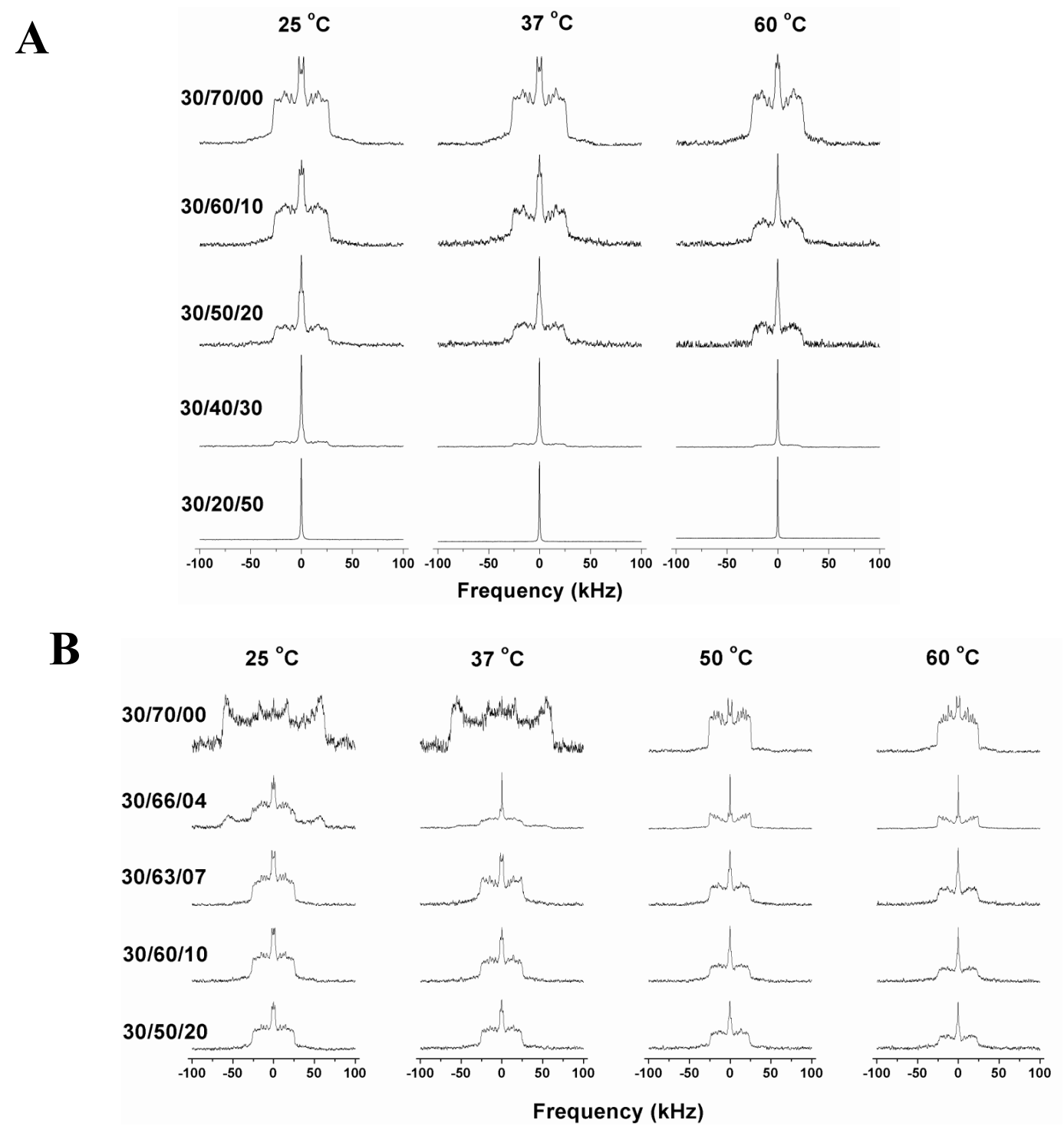

Figure 1. ${ }^{2} \mathrm{H}$ NMR spectra of PA- $d_{31} /$ Chol/PEG-Chol mixtures, $\mathrm{pH} 8.4(\mathrm{~A})$ and $5.0(\mathrm{~B})$.

Temperatures and molar ratios are indicated on the top of each column and on the left of each row, respectively.

The evolution of the proportion of isotropic phase in the ternary systems, as a function of PEG-Chol content is summarized in Figure 2. For PEG-Chol content lower than or equal to 20\%, all the fatty acid appeared to exist in the fluid lamellar phase. Larger proportions of PEG-Chol led to the formation of an isotropic phase that coexisted with the lo lamellar phase. The 
proportion of the isotropic phase steadily increased with the proportion of PEG-Chol. More than $85 \%$ of PA- $d_{31}$ existed in the isotropic phase for a PA- $d_{31} /$ Chol/PEG-Chol $30 / 20 / 50$ mixture. Higher temperatures favored the formation of the isotropic phase in the mixtures that included $30 \%$ or more of PEG-Chol (Figure 1A).

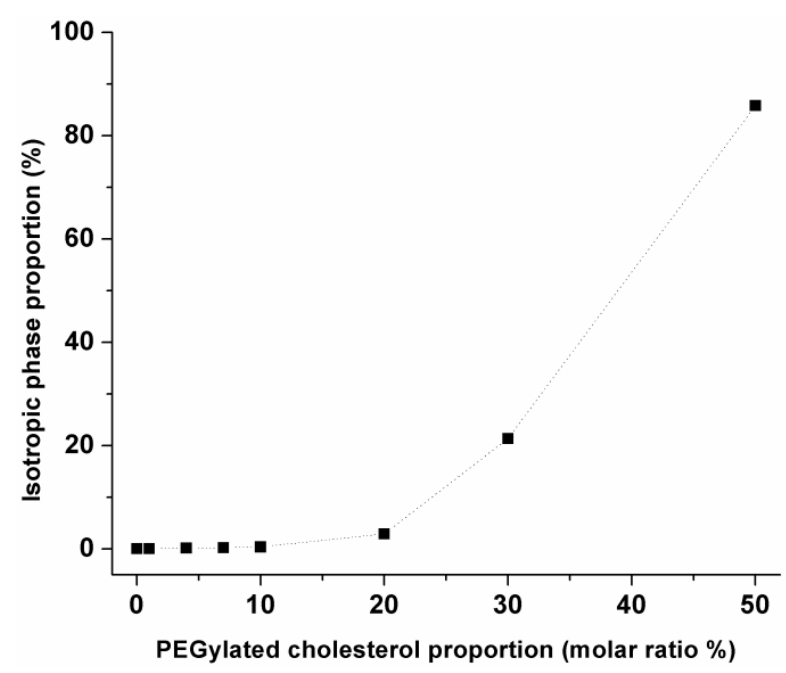

Figure 2. Dependence of the proportion of isotropic phase on the PEG-Chol content in PA- $d_{31} / \mathrm{Chol} / \mathrm{PEG}-\mathrm{Chol}$ mixtures, at $25^{\circ} \mathrm{C}$ and $\mathrm{pH}$ 8.4. The PA/sterol molar ratio remained 3/7.

It has been shown that a decrease of $\mathrm{pH}$ favors the formation of solid components in the $\mathrm{PA} / \mathrm{Chol}$ system, ${ }^{27,41}$ a property giving rise to $\mathrm{pH}$-sensitive formulations. In order to investigate the $\mathrm{pH}$ sensitivity of the PEG-containing mixtures, their spectra were recorded at $\mathrm{pH} 5.0$ (Figure 1B). At temperatures below $50{ }^{\circ} \mathrm{C}$, the spectra of a PA- $d_{31} /$ Chol $30 / 70$ mixture displayed only a signal typical of solid and immobile PA- $d_{31}$. At $50{ }^{\circ} \mathrm{C}$ and above, the solid signal completely disappeared and only a fluid lamellar component was observed, in agreement with previously reports. ${ }^{27,41}$ The widths of these spectra were found to be very similar to those observed at $\mathrm{pH} 8.4$ (Figure 1A), suggesting a comparable orientational order of the alkyl chain. The presence of PEG-Chol in the mixtures modified the phase behavior of the mixture and led to the formation of fluid bilayers at low temperatures (below $50{ }^{\circ} \mathrm{C}$ ). For example, at $25{ }^{\circ} \mathrm{C}$, the spectrum of 
PA- $d_{31} /$ Chol $(30 / 70)$ mixture indicated that the fatty acid was completely solid whereas it was completely in a fluid lamellar phase in the PA- $d_{31} / \mathrm{Chol} / \mathrm{PEG}-\mathrm{Chol} 30 / 63 / 07$ mixture. The co-existence of solid and fluid bilayers was observed for the mixtures containing $4 \%$ of PEG-Chol. The presence of PEG-Chol in the mixture downshifted the solid-to-fluid lamellar phase transition as the system containing 4\% PEG-Chol was completely fluid at $37{ }^{\circ} \mathrm{C}$ and that with 7\% PEG-Chol existed in the fluid lamellar phase over the whole investigated temperature range. At high temperatures and $\mathrm{pH} 8.4$, the presence of PEG-Chol has a limited effect on the structure of PA- $d_{31} / \mathrm{Chol} / \mathrm{PEG}-\mathrm{Chol}$ self-assemblies. The measured quadrupolar splittings were considerably large and similar to those obtained for the mixture without PEG-Chol in the same conditions; these ${ }^{2} \mathrm{H}$ NMR spectra indicated the formation of fluid lo bilayers. By promoting the formation of these fluid bilayers at low temperatures, PEG-Chol inhibited the $\mathrm{pH}$ sensitivity of the PA/Chol mixture; stable fluid bilayers were observed between $\mathrm{pH} 5.0$ and 8.4 when the system included between 7 and 20\% of PEG-Chol.

The ${ }^{2} \mathrm{H}$ NMR spectra provided a detailed characterization of the phase behavior from the fatty acid (PA- $\left.d_{31}\right)$ point of view. In order to examine the behavior of PEG-Chol in these systems, we measured the diffusion of PEG using pulsed-field-gradient ${ }^{1} \mathrm{H}$ NMR. This analysis is based on the assumption that it is possible to distinguish on the basis of the PEG diffusion coefficient between PEG-Chol inserted in 200-nm liposomes and PEG-Chol that would exist under a micellar form. Typically, there is one order of magnitude difference in the diffusion coefficient of micelles $\left(\sim 10^{-11} \mathrm{~m}^{2} / \mathrm{s}\right)$ and of liposomes $\left(\sim 10^{-12} \mathrm{~m}^{2} / \mathrm{s}\right) .{ }^{42}$ The diffusion coefficient was obtained from the PEG-Chol band at $\sim 3.66 \mathrm{ppm}$. For all the investigated PA- $d_{31} / \mathrm{Chol} / \mathrm{PEG}-\mathrm{Chol}$ mixtures (PEG-Chol proportions varying from 0 to 100), a single exponential could reproduce well the decay of the relative signal as a function of the gradient strength (see Eq. 1) and, consequently, a single diffusion coefficient was inferred (Figure 3). Pure PEG-Chol in $\mathrm{D}_{2} \mathrm{O}$ forms micelles. ${ }^{21,43}$ The measured diffusion coefficient in these conditions was $2.0 \times 10^{-11} \mathrm{~m}^{2} / \mathrm{s}$, a value 
corresponding to that reported for PEG2000-PE micelles. ${ }^{42}$ When PEG-Chol proportion in a mix with PA and cholesterol was between 1 and 20\%, PEG diffusion coefficients were relatively constant and corresponded to about $2.4 \times 10^{-12} \mathrm{~m}^{2} / \mathrm{s}$. This value was consistent with the diffusion coefficients reported for PEG2000-PE inserted in 100-nm phospholipid liposomes $\left(\sim 5 \times 10^{-12}\right.$ $\left.\mathrm{m}^{2} / \mathrm{s}\right){ }^{42}$ When the proportion of PEG-Chol was increased, the PEG diffusion coefficient also increased to reach, in the case of PA- $d_{31} / \mathrm{Chol} / \mathrm{PEG}-\mathrm{Chol} 30 / 20 / 50$ mixture, a value similar to that of pure PEG-Chol micelles in $\mathrm{D}_{2} \mathrm{O}$. These results showed that the presence of up to $20 \%$ of PEG-Chol in fluid bilayers made of PA and cholesterol had no significant influence on its diffusion coefficient. When its proportion was higher, the formation of smaller self-assemblies that diffused faster than liposomes was reported. This conclusion is in good agreement with the ${ }^{2} \mathrm{H}$ NMR results indicating the fast isotropic motions of PA- $d_{31}$ in mixtures where the proportion of PEG-Chol was higher than $20 \%$.

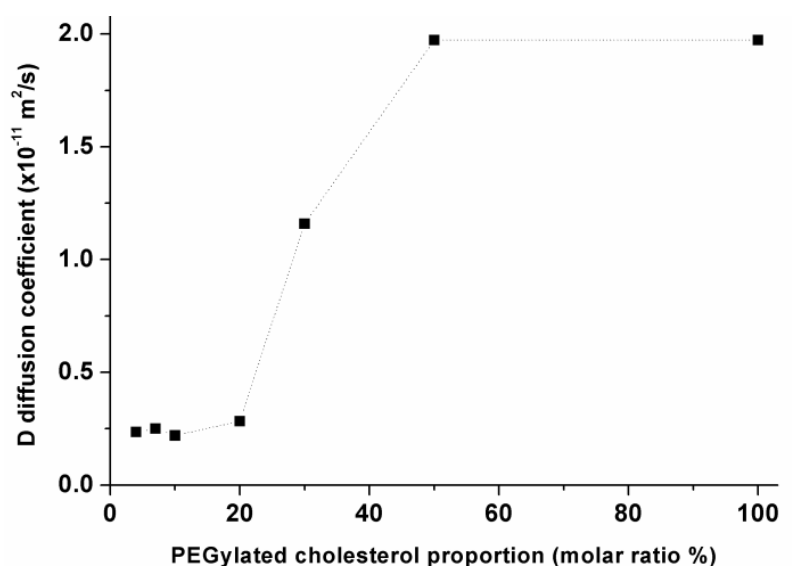

Figure 3. Evolution of the diffusion coefficient of PEG as a function of the PEG-Chol content in PA- $d_{31} / \mathrm{Chol} / \mathrm{PEG}-\mathrm{Chol} \mathrm{LUVs}$, at $25^{\circ} \mathrm{C}$ and $\mathrm{pH}$ 8.4. The PA/sterol ratio remained 3/7.

\section{Dynamic light scattering and Cryo-TEM}

It has been shown that, despite their very high cholesterol content, it is possible to extrude 
several mixtures of sterols and single-chain amphiphiles when they form a lo phase. ${ }^{27,29,44-45} \mathrm{We}$ examined the influence of PEG-Chol on the extrusion process of PA/Chol/PEG-Chol ternary systems. LUVs could be formed by extrusion of PA/Chol/PEG-Chol fluid bilayers with 30/66/04 and 30/60/10 molar ratios, between $\mathrm{pH} 5.5$ and 9.5. Typically a unimodal size distribution with an average diameter of about $100 \mathrm{~nm}$, consistent with the pore diameter of the polycarbonate filters $(100 \mathrm{~nm})$, was obtained. For example, the extrusion of PA/Chol/PEG-Chol (30/60/10) suspension at $\mathrm{pH} 7.4$, led to LUVs with an average diameter of $108 \pm 3 \mathrm{~nm}$, and a polydispersity index of $0.10 \pm 0.03$. PA/Chol/PEG-Chol LUVs were also examined by cryo-TEM (Figure 4). The impact of $\mathrm{pH}$ was studied by varying the external and internal $\mathrm{pH}$ to 8.4 or 5.0. The mixture containing 4\% PEG-Chol, with internal/external $\mathrm{pH} 8.4 / 8.4$ led predominantly to spherical vesicles of fairly uniform size. When both inside and outside $\mathrm{pH}$ of LUVs were 5.0, LUVs (Figure 4b) were also obtained but it seemed that they were less spherical than those formed at $\mathrm{pH}$ 8.4. The morphology of the vesicles prepared in the presence of $10 \mathrm{~mol} \%$ PEG-Chol were affected; although spherical and unilamellar vesicles dominated the samples, elongated, peanut-like, and convex (red blood cell like) shaped vesicles were observed for both $\mathrm{pH} 8.4 / 8.4$ and 5.0/5.0. 

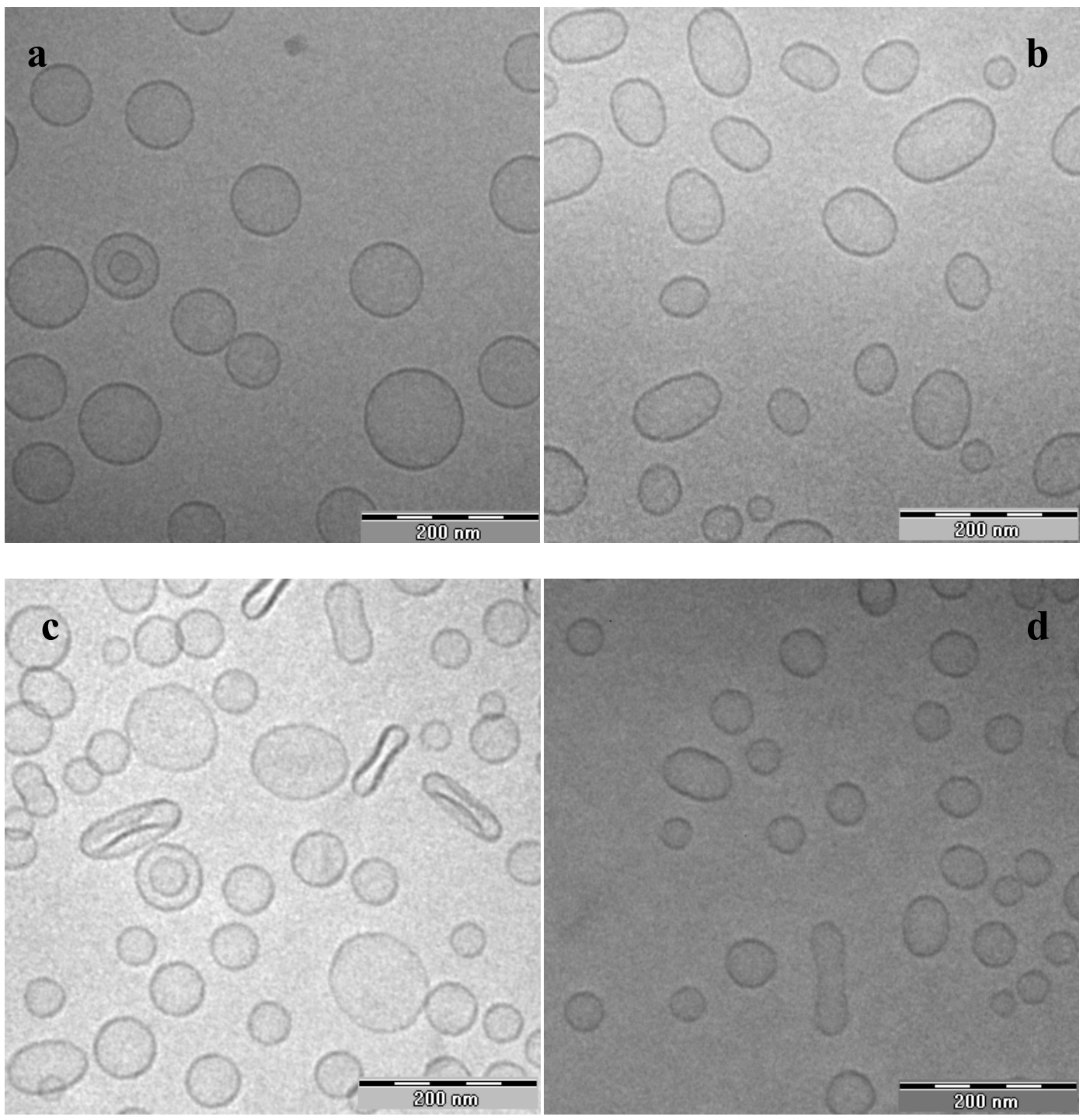

Figure 4. Cryo-micrographs of extruded PA/Chol/PEG-Chol LUVs in a molar ratio of 30/66/04 (a) pH 8.4/8.4 inside/outside, (b) $\mathrm{pH} 5.0 / 5.0$, and in a molar ratio of 30/60/10 (c) pH 8.4/8.4, (d) $\mathrm{pH} 5.0 / 5.0$. The scale bar corresponds to $200 \mathrm{~nm}$.

\section{PA/Chol/PEG-Chol LUV permeability}

The PA/Chol/PEG-Chol LUVs loaded with calcein were prepared by extrusion at $\mathrm{pH} 8.4$ as described above. The entrapped calcein showed an initial self-quenching of more than 0.90 , a 
value expected for an $80-\mathrm{mM}$ calcein solution. ${ }^{37}$. The passive release of calcein from these vesicles at $\mathrm{pH} 8.4$ was determined as a function of time (Figure 5). The leakage from PA/Chol/PEG-Chol LUVs (with a molar composition of 30/66/04 and 30/60/10) was very limited: even after three months, no significant release could be detected. This notable ability to retain the encapsulated calcein compares advantageously to other PEGylated liposomes ( ${ }^{46-47}$ for example).

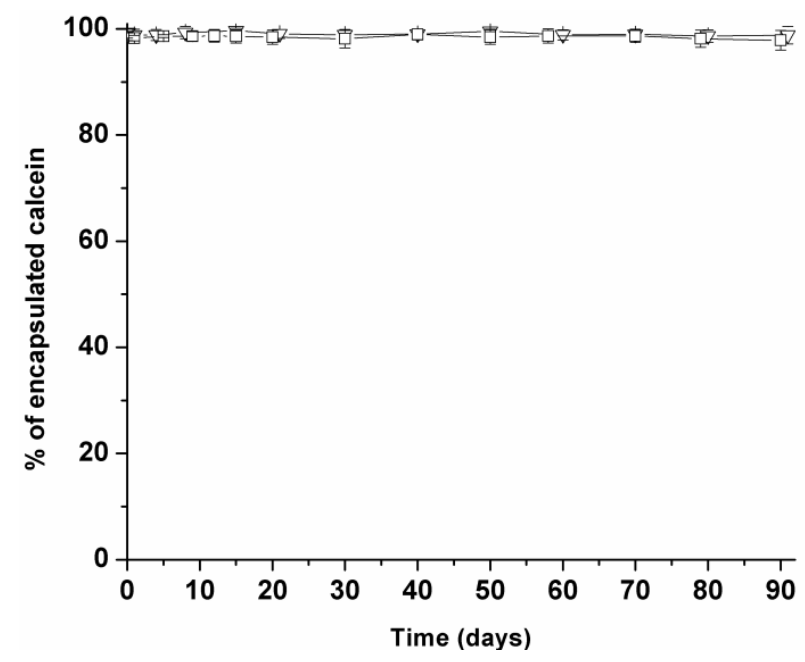

Figure 5. Passive leakage of entrapped calcein from LUVs prepared with mixtures of PA/Chol/PEG-Chol ( ) 30/66/04, and ( $\nabla$ ) 30/60/10 (molar ratio); the leakage was measured at room temperature, $\mathrm{pH} 8.4$.

The permeability of the PA/Chol/PEG-Chol LUVs was also assessed as a function of $\mathrm{pH}$ (Figure 6). As shown previously, ${ }^{29} \mathrm{PA} / \mathrm{Chol} \mathrm{LUVs}$ (30/70 molar ratio) are $\mathrm{pH}$-sensitive: they were stable between $\mathrm{pH} 7$ and 9.5 whereas a significant calcein release was observed for $\mathrm{pH} 6.0$ or below. Contrasting with this behavior, no significant calcein release was observed from the LUVs containing PEGylated cholesterol when the external $\mathrm{pH}$ was modified between 5.5 and 9.5. These results indicate that the presence of PEG-Chol in the LUVs impedes their $\mathrm{pH}$ sensitivity. 


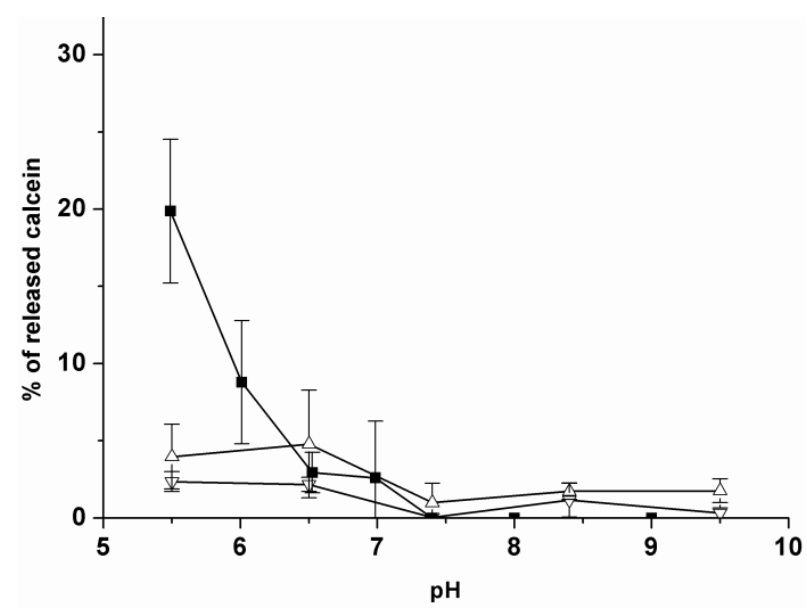

Figure 6. Effect of the external $\mathrm{pH}$ on the calcein release from (घ) PA/Chol/PEG-Chol 30/70/00, $(\Delta)$ 30/66/04, and $(\nabla)$ 30/60/10 LUVs. The measurements were carried out at room temperature, $\sim 24 \mathrm{~h}$ after the $\mathrm{pH}$ change.

\section{DOX Active Loading and Release}

The effectiveness of active loading using a transmembrane ammonium gradient was assessed for liposomes made of PA/Chol/PEG-Chol (30/60/10 molar ratio). The encapsulation efficiency of DOX was estimated to $84 \%$, and the resulting drug to lipid ratio was 0.06 (molar ratio). These parameters are comparable to those previously obtained with phospholipid-based liposomes. ${ }^{48-50}$ It should be pointed out that the reported trapping efficiency was very good since the drug to lipid ratio is expressed in molar terms and the investigated liposomes were formed from a single-chain amphiphile, in contrast to phospholipids, which bear two hydrophobic chains. The stability and the passive release of DOX-loaded liposomes were also examined. The results are summarized in Figure 7. It is shown that the non-phospholipid liposomes displayed a very limited permeability to DOX; the drug slowly leaked out from the LUVs and only $\sim 20 \%$ of encapsulated DOX was released after a three-month period. The stability of DOX-loaded PA/Chol/PEG-Chol LUVs was significantly improved compared to active-loaded 
phosphophatidylcholine liposome containing $38 \mathrm{~mol} \%$ of cholesterol and $5 \mathrm{~mol} \%$ of PEG-lipid (PEG-DSPE or PEG-Chol). For those formulations, more than $30 \%$ of the encapsulated DOX was released within three days, in similar conditions. ${ }^{23}$

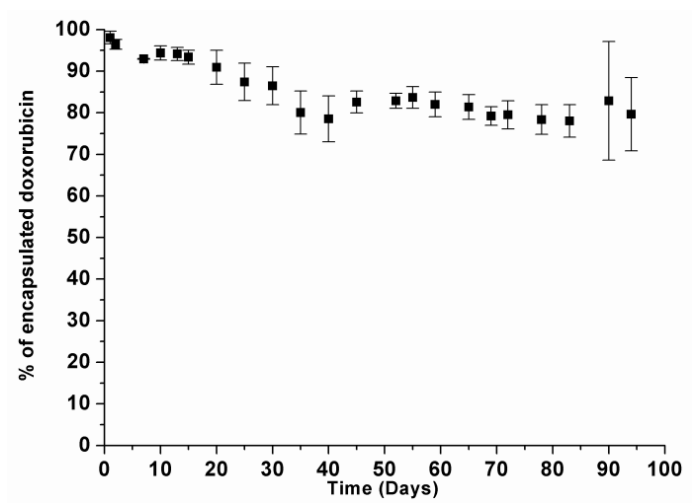

Figure 7. Passive leakage of actively loaded doxorubicin in PA/Chol/PEG-Chol 30/60/10 LUVs, measured at room temperature, $\mathrm{pH} 8.4$.

\section{Pharmaco-kinetics}

The circulation times of naked and PEGylated PA/Chol LUVs, as well as of DSPC/Chol/PEG-PE LUVs as a control, have been determined in rat blood system. The DiD fluorescence measurements (Figure 8) show that naked PA/Chol (30/70) LUVs were cleared very rapidly from the blood stream. After $0.16 \pm 0.05 \mathrm{~h}$, half the dose was still in the blood circulation. PA/Chol/PEG-Chol (30/60/10) LUVs displayed a significantly improved circulation time by a factor of $\sim 7$ : half the dose was present in the blood circulation after the injection $1.09 \pm 0.23 \mathrm{~h}$. Despite this enhancement, the LUV clearance was much faster than that observed for the control phospholipid cholesterol-containing PEGylated LUVs. For the DSPC/Chol/PEG-PE LUVs (55/45/5), 50\% of the dose was cleared after $17.5 \pm 2.0 \mathrm{~h}$, and $\sim 25 \%$ of the injected dose remained in the blood after $24 \mathrm{~h}$, a result consistent with previous studies ${ }^{51}$. The biodistribution after $24 \mathrm{~h}$ was determined for naked and PEGylated PA/Chol LUVs (Figure 9) (the DSPC/Chol/PEG-PE LUVs remaining in the blood stream after $24 \mathrm{~h}$ would have interfered with the measurements, increasing the apparent distribution in the highly vascularized organs). 
As expected, most of DiD was found in the liver for both formulations, indicative of the MPS uptake. ${ }^{51}$ A significant quantity was also found in the spleen for PA/Chol LUVs. It appears that the presence of PEG at the interface has affected the biodistribution as, in addition to liver, a significant amount of DiD was also found in the lungs and the heart whereas less dye was found in the spleen, a phenomenon previously observed upon PEGylation of liposomes. ${ }^{9}$

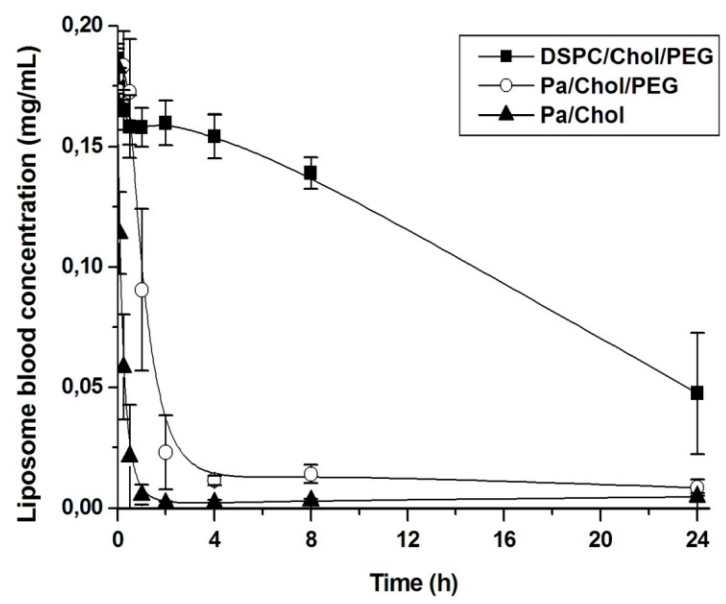

Figure 8. Pharmaco-kinetics of the blood clearance of DiD-labeled LUVs after intravenous administration in rats; (4): PA/Chol (30/70) LUVs, (०) PA/Chol/PEG-Chol (30/60/10) LUVs, (-) control DSPC/Chol/PEG-PE (55/45/5) LUVs ( $\mathrm{n}=8$, the average values are presented and the error bars represent the standard deviations). 


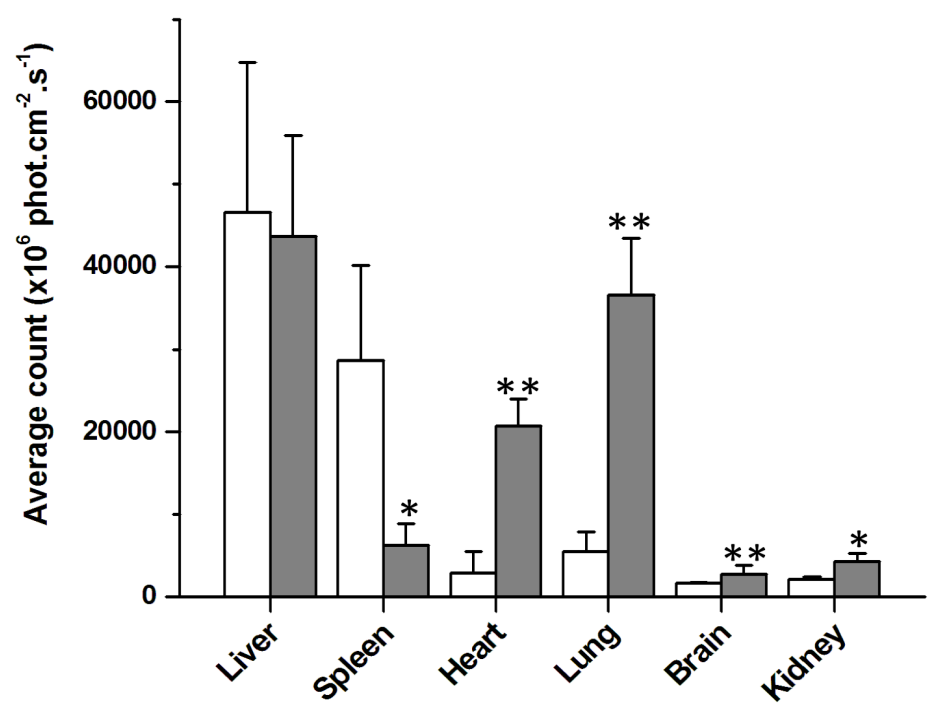

Figure 9. DiD-labeled LUV biodistribution in rats after 24 h; white bars: PA/Chol (30/70) LUVs, black bars PA/Chol/PEG-Chol (30/60/10) LUVs, $(n=8$, the average values are presented and the error bars represent the standard deviations). Significant differences in the organ accumulation based on the Mann Whitney test: * p $\leq 0.003, \quad * * \mathrm{p} \leq 0.0003$ )

\section{Discussion}

The present study reveals that it is possible to introduce PEGylated cholesterol into liposomes prepared from palmitic acid and cholesterol. At least $10 \mathrm{~mol} \%$ of PEG-Chol can be included in the ternary system before non-liposomal structures start to appear. ${ }^{2} \mathrm{H}$ NMR results show that all the palmitic acid molecules are included in the fluid lamellar phase while the diffusion coefficient of PEG-Chol indicates that PEG-Chol molecules are anchored to the liposomes. Moreover the cryo-EM analysis showed that spherical unilamellar vesicles dominated the samples for the PA/Chol mixtures that included 4 and 10 mol\% of PEG-Chol. With $10 \mathrm{~mol} \%$ 
PEG-Chol, different morphologies such as elongated, dumbbell-like and erythrocyte-like shapes were observed occasionally. As discussed elsewhere ${ }^{43,52}$, the occurrence of these shapes could be associated with a partial component segregation in the vesicles leading to a cholesterol content somewhat lower in the highly curved "ends" of the vesicles while PEG-Chol is enriched in these regions, reducing the interPEG steric repulsion. Beyond $20 \mathrm{~mol} \%$ of PEG-Chol, an isotropic phase is formed (Figure 2, looking at PA- $d_{31}$ ) and the diffusion coefficient of PEG-Chol increases to a value similar to that obtained for PEG-Chol micelles (Figure 3). It has been shown that an increasing proportion of PEG-lipid in bilayers of several systems leads to a progressive transformation of liposomes. There is initially the formation of open-bilayer fragments and disks that decrease in size with higher concentration of PEG-lipids to become indistinguishable from PEG-lipid micelles. ${ }^{52-53}$ Therefore, the evolution of the NMR signals is likely due to the formation of mixed micelles of PEG-Chol with PA, and cholesterol. On the basis of the PFG NMR measurements, it appears that the bilayer solubilization is complete and only micellar-like forms are obtained when the mixture contains about $50 \%$ of PEG-Chol. The observation of disks and bilayers fragments has been reported upon the inclusion of PEG-lipid in phospholipid bilayers. As discussed in detail elsewhere, ${ }^{43,52,54-55}$ this morphology transition was observed for a PEG-lipid content varying between 7 and $20 \mathrm{~mol} \%$, depending on several parameters including the molecular weight of PEG, and the composition of the bilayers. Because the NMR data and the cryo-TEM images confirmed that $10 \mathrm{~mol} \%$ of PEG-Chol can be inserted in PA/Chol liposomes without significant morphological changes, we have focused our characterization of permeability and of pharmaco-kinetics on liposomes formed by PA/Chol/PEG-Chol mixtures with a PEG-Chol content of $10 \mathrm{~mol} \%$ or less.

It has been shown that $\mathrm{PA} / \mathrm{Chol}$ system forms stable fluid bilayers only at high $\mathrm{pH}(\geq 7.5)$ while lower $\mathrm{pH}$ favors the formation of solid phase cholesterol and palmitic acid. ${ }^{41}$ The protonation state of the carboxylic group dictates the bilayer stability through its modulation of 
the lipid mixing properties and the bilayer interface hydration. At lower $\mathrm{pH}, \mathrm{PA}$ head group is protonated, favoring phase separation from cholesterol and reducing the hydration of the bilayer interface; as a consequence, the destabilization of the fluid lamellar phase is observed. ${ }^{28,45}$ In the present work, it is shown that the incorporation of PEG-Chol favors the formation of a lo phase at lower $\mathrm{pH}$. For example, typical spectrum of cholesterol inserted in fluid bilayers was obtained at $\mathrm{pH} 5,25^{\circ} \mathrm{C}$, when the PA-d $\mathrm{d}_{31} /$ Chol system included $7 \mathrm{~mol} \%$ PEG-Chol (Figure 1B). It has been proposed that interfacial PEG may hinder the protonation of carboxylic groups located at the interface. ${ }^{56}$ Consequently, a larger proportion of PA may be unprotonated in PEGylated liposomes, favoring lamellar phases, and, as a consequence, a reduced pH-sensitivity. It is also possible that the PEGylation of cholesterol leads to steric repulsion of the modified cholesterol molecules in the plane of the bilayer, thus promoting mixing and formation of a homogenous lo phase. The presence of PEG hydrophilic chains at the bilayer interface maintaining a suitable degree of hydration of the bilayer, and the strong positive spontaneous curvature of PEG-lipids may also play a role in the hindrance of the $\mathrm{pH}$-induced phase transition. The promotion of a fluid bilayer phase by PEG-Chol makes possible the extrusion of the ternary mixtures to form LUVs, even at low $\mathrm{pH}$ such as 5.0; this operation was proved impossible for PA/Chol system at such low $\mathrm{pH}^{45}$

PEG-Chol-containing non-phospholipid liposomes display a limited permeability compared to that generally observed for PEGylated phospholipid liposomes, as is shown in this paper for calcein as well as for DOX. This distinct impermeability is consistent with that previously reported for other similar systems prepared from a single-chain amphiphile and a sterol. $^{27,29}$ This property has been associated with the high content of cholesterol leading to an increase in acyl chain order, ${ }^{40,57}$ and a decreased permeability. The insertion of PEGylated cholesterol in the PA/Chol bilayers does not appear to have a significant effect on the passive permeability of the resulting LUVs. This observation is in good agreement with the absence of 
influence of PEG-Chol on the quadrupolar splittings of PA- $d_{31}$ in the ${ }^{2} \mathrm{H}$ NMR spectra of PA- $d_{31} / \mathrm{Chol} / \mathrm{PEG}-\mathrm{Chol}$ systems, the splittings being representative of the alkyl chain orientational order.

It was shown that the content release from $\mathrm{PA} / \mathrm{Chol}$ liposomes could be triggered by a $\mathrm{pH}$ variation $^{28-29} ; \mathrm{pH} \leq 7.5$ would lead to the formation of solid PA as well as solid cholesterol, causing the complete disruption of the liposomes and as a consequence, the complete release of their content. This $\mathrm{pH}$-stimulated release is however significantly reduced for the PEG-coated non-phospholipid liposomes. Actually, practically no release can be triggered by decreasing the external $\mathrm{pH}$ to 5.5 , in contrast to a $\mathrm{pH}$-triggered release of about $20 \%$ for the naked $\mathrm{PA} / \mathrm{Chol}$ liposomes (Figure 6). This result is consistent with the impact of interfacial PEG on the phase behavior of PA/Chol mixtures. As discussed above, the presence of interfacial PEG promotes the formation of a fluid lamellar phase at $\mathrm{pH}$ as low as 5 . As a consequence, the solid-fluid transition observed for the $\mathrm{PA} / \mathrm{Chol}$ system during a $\mathrm{pH}$ variation from 5 to 8.4 is no longer detected in the presence of $10 \mathrm{~mol} \% \mathrm{PEG}$; the system remains in the fluid lamellar phase over the whole $\mathrm{pH}$ range and, as a consequence, their liposomes remain stable and impermeable. The decrease of $\mathrm{pH}$ sensitivity appears to be commonly observed for PEG-coated phospholipid liposomes. For example, the inclusion of 1 to $5 \mathrm{~mol} \%$ PEG-DSPE in pH-sensitive DOPE/oleic acid liposomes shifted the $\mathrm{pH}$-trigged release curve towards more acidic regions and reduced the extent of the leakage that could be induced. ${ }^{56}$ The presence of only $1 \mathrm{~mol} \%$ PEG-DSPE in the formulation led to a decrease of release from $>90 \%$ without PEG to $\sim 20 \%$, when the $\mathrm{pH}$ was set to 5 . Similarly the $\mathrm{pH}$-sensitivity of cholesteryl hemisuccinate/DOPE liposomes was attenuated in the presence of PEG-PE: ${ }^{58}$ the nearly complete release of the liposome content could be trigged at $\mathrm{pH} 5$, while the inclusion of $2 \mathrm{~mol} \%$ PEG-PE reduced the $\mathrm{pH}$-induced leakage to $\sim 20 \%$. Therefore, the presence of interfacial PEG changes the chemistry of the bilayer/aqueous milieu interface and attenuates the $\mathrm{pH}$-response of functional groups such as the carboxylic group, leading to the 
reduction or even the loss of $\mathrm{pH}$-responsiveness of PEGylated liposomes.

We also determined the performance of PA/Chol/PEG-Chol liposomes for active loading of DOX. It is shown that DOX could be successfully loaded in the aqueous compartment of these LUVs with a conventional approach based on an ammonium sulfate gradient. A high drug loading efficiency (84\%) and a high drug to lipid ratio (0.06) were obtained, despite the much reduced bilayer permeability of these liposomes and the presence of interfacial PEG. The reported values are comparable to those reported for phospholipid liposomes. ${ }^{33}$

Finally, the pharmaco-kinetic results indicate that PA/Chol/PEG-Chol LUVs injected intravenously display an extended circulation time in the blood stream compared to naked ones but they are cleared more rapidly than DSPC/Chol/PEG-PE liposomes. The circulation lifetime depends on several parameters ${ }^{10-11}$ and its predictability is, with our current knowledge, limited. The presence of interfacial PEG increases the circulation time of PA/Chol LUVs as expected; the PEGylated LUVs remain in the blood stream considerably longer than the naked ones (a half-life time that is 6 times longer). In addition, a reduced amount of dye was found in the spleen when LUVs were PEGylated, another indication of a limited uptake by the MRS. A tight chain packing in the hydrophobic core of bilayers has been shown to extend the circulation time of liposomes, among other, by inhibiting the binding of plasma proteins. ${ }^{5,10}{ }^{2} \mathrm{H}-\mathrm{NMR}$ results indicate that the orientational order of PA acyl chain is very high, likely because of the large cholesterol content. The combined features of interfacial PEG and highly ordered bilayer core are predictive promoters of extended circulation time in the blood. However, several negatively charged lipids were shown to markedly shorten the liposome clearance kinetics. ${ }^{10,59}$ This phenomenon was associated to a higher binding affinity of these anionic liposomes for blood proteins. It appears that this unfavorable electrostatic factor dominates the others in determining the lifetime of PA/Chol/PEG-Chol liposomes in the blood circulation and leads to a relatively 
rapid clearance. The presence of interfacial charges is a common prerequisite for the formation of stable liposomes from mixtures of single-chain amphiphile and sterol because it favors lipid mixing and ensures the proper hydration of the self-assembly interface ${ }^{25}$. Nevertheless, it has been shown that all anionic lipids do not have the same impact on liposomes clearance: ${ }^{10,59}$ some anionic phospholipid, including phosphatidylglycerol, phosphatidylinositol, and synthetic anionic acylated phosphatidylethanolamines, actually improve circulation lifetime in blood. Therefore additional investigations may identify more suitable chemical compositions of liposomes made of single-chain amphiphile and sterol (by incorporating, for example, anionic cholesterol sulphate such as in ref ${ }^{60}$ ) in order to create new liposomes with extended circulation time in the blood while maintaining the reduced permeability associated with its high sterol content.

\section{Conclusions}

The present study identifies several characteristics of novel non-phospholipid liposomes made of PA/Chol/PEG-Chol mixtures. It is shown that up to $20 \mathrm{~mol} \%$ of PEG-Chol can be introduced in these non-phospholipid sterol-rich mixtures without disturbing the lamellar structure. The presence of PEG, anchored using a cholesterol moiety, makes PA/Chol/PEG-Chol liposomes stable between $\mathrm{pH} 5$ and 9.5. A very low permeability was obtained for encapsulated calcein and DOX. Active loading of DOX can be achieved with this ternary system. The pharmaco-kinetics investigations of the circulation lifetime, and the biodistribution indicate that, despite the interfacial PEG and the tight chain packing of the bilayer core, these nanovectors are cleared relatively rapidly from the blood circulation, a phenomenon likely associated with the presence of negatively charged unprotonated PA. The relationships between different properties of these self-assemblies with uncommon chemical composition are useful in orienting the development of superior nanovectors. 


\section{Acknowledgement}

The authors thank the Natural Sciences and Engineering Research Council of Canada, the Fonds Québécois de la Recherche sur la Nature et les Technologies, the Scientific Council of Angers University, the Swedish Research Council, and the Swedish Cancer Society for the financial support. Z.-K. C is grateful to Université de Montréal and China Scholarship Council for his scholarship. We thank Cédric Malveau (Department of Chemistry, Université de Montréal), for his technical support during the NMR experiments, Göran Karlsson (Chemistry Department, Uppsala University), for carrying out the cryo-TEM analysis, and Pierre Legras (SCAHU, Angers) for the animal experiments. 


\section{References}

1. Sawant, R. R.; Torchilin, V. P. Liposomes as 'smart' pharmaceutical nanocarriers. Soft Matter 2010, 6, 4026-4044.

2. Immordino, M. L.; Dosio, F.; Cattel, L. Stealth liposomes: review of the basic science, rationale, and clinical applications, existing and potential. Int. J. Nanomed. 2006, 1, 297-315.

3. Damen, J.; Regts, J.; Scherphof, G. Transfer and exchange of phospholipid between small unilamellar liposomes and rat plasma high density lipoproteins Dependence on cholesterol content and phospholipid composition. Biochim. Biophys. Acta 1981, 665, 538-545.

4. Patel, H. M.; Tuzel, N. S.; Ryman, B. E. Inhibitory effect of cholesterol on the uptake of liposomes by liver and spleen Biochim. Biophys. Acta 1983, 761, 142-151.

5. Semple, S. C.; Chonn, A.; Cullis, P. R. Influence of cholesterol on the association of plasma proteins with liposomes. Biochemistry 1996, 35, 2521-2525.

6. Gabizon, A.; Papahadjopoulos, D. Liposome formulations with prolonged circulation time in blood and enhanced uptake by tumors. Proc. Natl. Acad. Sci. U. S. A. 1988, 85, 6949-6953.

7. Allen, T. M.; Hansen, C.; Rutledge, J. Liposomes with prolonged circulation times: factors affecting uptake by reticuloendothelial and other tissues. Biochim. Biophys. Acta 1989, 981, 27-35.

8. Maruyama, K.; Yuda, T.; Okamoto, A.; Ishikura, C.; Kojima, S.; Iwatsuru, M. Effect of molecular weight in amphipathic polyethyleneglycol on prolonging the circulation time of large unilamellar liposomes. Chem. Pharm. Bull. 1991, 39, 1620-1622.

9. Allen, T. M.; Hansen, C.; Martin, F.; Redemann, C.; Yau-Young, A. Liposomes containing synthetic lipid derivatives of poly(ethylene glycol) show prolonged circulation half-lives in vivo. Biochim. Biophys. Acta 1991, 1066, 29-36.

10. Drummond, D. C.; Noble, C. O.; Hayes, M. E.; Park, J. W.; Kirpotin, D. B. Pharmacokinetics and in vivo drug release rates in liposomal nanocarrier development. J. Pharm. Sci. 2008, 97, 4696-4740.

11. Drummond, D. C.; Meyer, O.; Hong, K.; Kirpotin, D. B.; Papahadjopoulos, D. Optimizing liposomes for delivery of chemotheraputic agents to solid tumors. Pharm. Rev. 1999, 51, 691-743.

12. Allen, T. M. The use of glycolipids and hydrophilic polymers in avoiding rapid uptake of liposomes by the mononuclear phagocyte system. Adv. Drug Delivery Rev. 1994, 13, 285-309. 
13. Bedu-Addo, F. K.; Huang, L. Interaction of PEG-phospholipid conjugates with phospholipid: implications in liposomal drug delivery. Adv. Drug Delivery Rev. 1995, 16, 235-247.

14. Du, H.; Chandaroy, P.; Hui, S. W. Grafted poly-(ethylen glycol) on lipid surfaces inhibits protein adsorption and cell adhesion. Biochim. Biophys. Acta 1997, 1326, 236-248.

15. Lasic, D. D.; Martin, F. J.; Gabizon, A.; Huang, S. K.; Papahadjopoulos, D. Sterically stabilized liposomes: a hypothesis on the molecular origin of the extended circulation times. Biochim. Biophys. Acta 1991, 1070, 187-192

16. Dos Santos, N.; Allen, C.; Doppen, A.-M.; Anantha, M.; Cox, K. A. K.; Gallagher, R. C.; Karlsson, G.; Edwards, K.; Kenner, G.; Samuels, L.; Webb, M. S.; Bally, M. B. Influence of poly(ethylene glycol) grafting density and polymer length on liposomes: Relating plasma circulation lifetimes to protein binding. Biochim. Biophys. Acta 2007, 1768, 1367-1377.

17. Wong, B.; Boyer, C.; Steinbeck, C.; Peters, D.; Schmidt, J.; van Zanten, R.; Chmelka, B.; Zasadzinski, J. A. Design and in situ characterization of lipid containers with enhanced drug retention. Adv. Mater. 2011, 23, 2320-2325.

18. Semple, S. C.; Chonn, A.; Cullis, P. R. Interactions of liposomes and lipid-based carrier systems with blood proteins: Relation to clearance behaviour in vivo. Adv. Drug Delivery Rev. 1998, 32, 3-17.

19. Bedu-Addo, F. K.; Tang, P.; Xu, Y.; Huang, L. Effects of polyethyleneglycol chain length and phospholipid acyl chain composition on the interaction of polyethyleneglycol-phospholipid conjugates with phospholipid: Implications in liposomal drug delivery. Pharm. Res. 1996, 13, 710-717.

20. Webb, M. S.; Saxon, D.; Wong, F. M. P.; Lim, H. J.; Wang, Z.; Bally, M. B.; Choi, L. S. L.; Cullis, P. R.; Mayer, L. D. Comparison of different hydrophobic anchors conjugated to poly(ethylene glycol): effects on the pharmacokinetics of liposomal vincristine. Biochim. Biophys. Acta 1998, 1372, 272-282.

21. Ishiwata, H.; Vertut-Doï, A.; Hirose, T.; Miyajima, K. Physical-chemistry characteristics and biodistribution of poly(ethylene glycol)-coated liposomes using poly(oxyethylene) cholesteryl ether. Chem. Pharm. Bull. 1995, 43, 1005-1011.

22. VertutDoi, A.; Ishiwata, H.; Miyajima, K. Binding and uptake of liposomes containing a poly(ethylene glycol) derivative of cholesterol (stealth liposomes) by the macrophage cell line J774: Influence of PEG content and its molecular weight. Biochim. Biophys. Acta 1996, 1278, 19-28.

23. Zhao, X. B.; Muthusamy, N.; Byrd, J. C.; Lee, R. J. Cholesterol as a bilayer anchor for 
PEGylation and targeting ligand in folate-receptor-targeted liposornes. J. Pharm. Sci. 2007, 96, 2424-2435.

24. Sant, V. P.; Nagarsenker, M. S. Synthesis of Monomethoxypolyethyleneglycol-Cholesteryl Ester and Effect of its Incorporation in Liposomes. AAPS PharmSciTech 2011, 12, 1056-1063.

25. Cui, Z.-K.; Lafleur, M. Lamellar self-assemblies of single-chain amphiphiles and sterols and their derived liposomes: distinct compositions and distinct properties. Colloids Surf., B 2014, 114, $117-185$.

26. Marianecci, C.; Di Marzio, L.; Rinaldi, F.; Celia, C.; Paolino, D.; Alhaique, F.; Esposito, S.; Carafa, M. Niosomes from 80s to present: the state of the art. Adv. Colloid Interface Sci. 2014, 205, 187-206.

27. Cui, Z.-K.; Bastiat, G.; Jin, C.; Keyvanloo, A.; Lafleur, M. Influence of the nature of the sterol on the behavior of palmitic acid/sterol mixtures and their derived liposomes. Biochim. Biophys. Acta 2010, 1798, 1144-1152.

28. Phoeung, T.; Aubron, P.; Rydzek, G.; Lafleur, M. pH-triggered release from nonphospholipid LUVs modulated by the pKa of the included fatty acid. Langmuir 2010, 26, 12769-12776.

29. Bastiat, G.; Oliger, P.; Karlsson, G.; Edwards, K.; Lafleur, M. Development of non-phospholipid liposomes containing a high cholesterol concentration. Langmuir 2007, 23, 7695-7699.

30. Thomas, A. M.; Kapanen, A. I.; Hare, J. I.; Ramsay, E.; Edwards, K.; Karlsson, G.; Bally, M. B. Development of a liposomal nanoparticle formulation of 5-Fluorouracil for parenteral administration: Formulation design, pharmacokinetics and efficacy. J. Controlled Release 2011, $150,212-219$.

31. Harrington, K. J.; Syrigos, K. N.; Vile, R. G. Uposomally targeted cytotoxic drugs for the treatment of cancer. J. Pharm. Pharmacol. 2002, 54, 1573-1600.

32. Mayer, L. D.; Tai, L. C. L.; Ko, D. S. C.; Masin, D.; Ginsberg, R. S.; Cullis, P. R.; Bally, M. $\mathrm{B}$. Influence of vesicle size, lipid composition, and drug-to-lipid ratio on the biological-activity of liposomal doxorubicin in mice Cancer Res. 1989, 49, 5922-5930.

33. Haran, G.; Cohen, R.; Bar, L. K.; Barenholz, Y. Transmembrane ammonium sulfate gradients in liposomes produce efficient and stable entrapment of amphipathic weak bases. Biochim. Biophys. Acta 1993, 1151, 201-215.

34. Bastiat, G.; Pritz, C. O.; Roider, C.; Fouchet, F.; Lignières, E.; Jesacher, A.; Glueckert, R.; 
Ritsch-Marte, M.; Schrott-Fisher, A.; Saulnier, P.; Benoit, J. P. A new tool to ensure the fluorescent dye labeling stability of nanocarriers: a real challenge for fluorescence imaging. $J$. Controlled Release 2013, 170, 334-342.

35. Tanner, J. E. Use of the stimulated echo in NMR diffusion studies. J. Chem. Phys. 1970, 52, 2523-2526.

36. Almgren, M.; Edwards, K.; Karlsson, G. Cryo transmission electron microscopy of liposomes and related structures. Colloids Surf., A 2000, 174, 3-21.

37. Allen, T. M. Calcein as a tool in liposome methodology. In Liposome Technology, Gregoriadis, G., Ed.; CRC Press: Boca Raton, FC, 1984, pp 177-182.

38. Calvo, P.; Gouritin, B.; Chacun, H.; Desmaële, D.; D’Angelo, J.; Noel, J. P.; Georgin, D.; Fattal, E.; Andreux, J. P.; Couvreur, P. Long-circulating PEGylated polycyanoacrylate nanoparticles as new drug carrier for brain delivery. Pharm. Res. 2001, 18, 1157-1166.

39. Paré, C.; Lafleur, M. Formation of liquid ordered lamellar phases in the palmitic acid/cholesterol system. Langmuir 2001, 17, 5587-5594.

40. Vist, M. R.; Davis, J. H. Phase equilibria of cholesterol/dipalmitoylphosphatidylcholine mixtures: ${ }^{2} \mathrm{H}$ nuclear magnetic resonance and differential scanning calorimetry. Biochemistry 1990, 29, 451-464.

41. Ouimet, J.; Croft, S.; Paré, C.; Katsaras, J.; Lafleur, M. Modulation of the polymorphism of the palmitic acid/cholesterol system by the pH. Langmuir 2003, 19, 1089-1097.

42. Leal, C.; Rögnvaldsson, S.; Fossheim, S.; Nilssen, E. A.; Topgaard, D. Dynamic and structural aspects of PEGylated liposomes monitored by NMR. J. Colloid Interface Sci. 2008, 325, 485-493.

43. Beugin, S.; Edwards, K.; Karlsson, G.; Ollivon, M.; Lesieur, S. New sterically stabilized vesicles based on nonionic surfactant, cholesterol, and poly(ethylene glycol)-cholesterol conjugates. Biophys. J. 1998, 74, 3198-3210.

44. Phoeung, T.; Morfin Huber, L.; Lafleur, M. Cationic detergent/sterol mixtures can form fluid lamellar phases and stable unilamellar vesicles. Langmuir 2009, 25, 5778-5784.

45. Bastiat, G.; Lafleur, M. Phase behavior of palmitic acid/cholesterol/cholesterol sulfate mixtures and properties of the derived liposomes. J. Phys. Chem. B 2007, 111, 10929-10937.

46. Carrion, C.; Domingo, J. C.; de Madariaga, M. A. Preparation of long-circulating immunoliposomes using PEG-cholesterol conjugates: effect of the spacer arm between PEG and cholesterol on liposomal characteristic. Chem. Phys. Lipids 2001, 113, 97-110. 
47. Silvander, M.; Johnsson, M.; Edwards, K. Effects of PEG-lipids on permeability of phosphatidylcholine/cholesterol liposomes in buffer and in human serum. Chem. Phys. Lipids 1998, $97,15-26$.

48. Fritze, A.; Hens, F.; Kimpfler, A.; Schubert, R.; Peschka-Suss, R. Remote loading of doxorubicin into liposomes driven by a transmembrane phosphate gradient. Biochim. Biophys. Acta 2006, 1758, 1633-1640.

49. Mayer, L. D.; Bally, M. B.; Cullis, P. R. Strategies for optimizing liposomal doxorubicin. J. Liposome Res. 1990, 1, 463-480.

50. Amselem, S.; Cohen, R.; Druckmann, S.; Gabizon, A.; Goren, D.; Abra, R. M.; Huang, A.; New, R.; Barenholz, Y. Preparation and characterization of liposomal doxorubicin for human use. J. Liposome Res. 1992, 2, 93-123.

51. Woodle, M. C.; Collins, L. R.; Sponsler, E.; Kossovsky, N.; Papahadjopoulos, D.; Martin, F. J. Sterically stabilized liposomes. Reduction in electrophoretic mobility but not electrostatic surface potential. Biophys. J. 1992, 61, 902-910.

52. Edwards, K.; Johnsson, M.; Karlsson, G.; Silvander, M. Effect of polyethyleneglycol-phospholipids on aggregate structure in preparations of small unilamellar liposomes. Biophys. J. 1997, 73, 258-266.

53. Johansson, E.; Engvall, C.; Arfvidsson, M.; Lundahl, P.; Edwards, K. Development and initial evaluation of PEG-stabilized bilayers disks as novel model membranes. Biophys. Chem. 2005, 113, 183-192.

54. Johnsson, M.; Edwards, K. Liposomes, disks, and spherical micelles: aggregate structure in mixtures of gel phase phosphatidylcholines and poly(ethylene glycol)-phospholipids. Biophys. $J$. 2003, $85,3839-3847$.

55. Sandström, M. C.; Johansson, E.; Edwards, K. Structure of mixed micelles formed in PEG-lipid/lipid dispersions. Langmuir 2007, 23, 4192-4198.

56. Hong, M. S.; Lim, S. J.; Oh, Y. K.; Kim, C. K. pH-sensitive, serum-stable and long-circulating liposomes as a new drug delivery system. J. Pharm. Pharmacol. 2002, 54, 51-58.

57. Bloom, M.; Evans, E.; Mouritsen, O. G. Physical properties of the fluid lipid-bilayer component of cell membranes: a perspective. Q. Rev. Biophys. 1991, 24, 293-397.

58. Slepushkin, V. A.; Simoes, S.; Dazin, P.; Newman, M. S.; Guo, L. S.; Pedroso de Lima, M. C.; Duzgunes, N. Sterically stabilized pH-sensitive liposomes - Intracellular delivery of aqueous contents and prolonged circulation in vivo. J. Biol. Chem. 1997, 272, 2382-2388. 
59. Chonn, A.; Semple, S. C.; Cullis, P. R. Association of blood proteins with large unilamellar liposomes in vivo. J. Biol. Chem. 1992, 267, 18759-18765.

60. Carbajal, G.; Cui, Z.-K.; Lafleur, M. Non-phospholipid liposomes with a sterol content display a very limited permeability. Science China Chemistry 2013, 56, 40-47. 